\section{Tracheotomie Neugeborener - bald noch seltener nötig?}

\author{
Eine Tracheotomie bei Kindern ist etwas Besonderes. Aber jede größere \\ Kinderklinik und zahlreiche niedergelassene Kinderärzte betreuen durchaus \\ Kinder, die durch ein Tracheostoma atmen oder beatmet werden. Zwei \\ aktuelle Beiträge im Journal of Pediatrics widmen sich diesem Thema.
}

W ie häufig und warum werden Neugeborene überhaupt einer Tracheotomie unterzogen? Wie lässt sich zumindest für die Untergruppe der „lungenkranken“ Kinder durch protektive Therapieformen eine weitere Organschädigung mit der gelegentlichen Konsequenz eines operativen Luftröhrenschnittes weiter verringern? Zur Beantwortung dieser Fragen wurden fast 900.000 Neugebore von 348 Neugeborenen-Intensivabteilungen Nordamerikas im Zeitraum zwischen 1997 und 2012 gescreent. Es wurden 885 Patienten identifiziert, die innerhalb des 27. bis 119. Lebenstages tracheotomiert worden waren [1]. In diese elektronische Auswertung von Krankenberichten flossen gut $70 \%$ Frühgeborene jeglichen postmenstruellen Alters bei $\mathrm{Ge}$ burt ein.

So tauchte wenig überraschend auch die bronchopulmonale Dysplasie mit $45 \%$ neben oberen Atemwegsanomalien (23\%) und Larynxpathologien (13\%) als häufigste mit ursächliche Assoziation auf. Die „in hospital“-Mortalität lag bei $14 \%$, hiervon waren vor allem Kinder

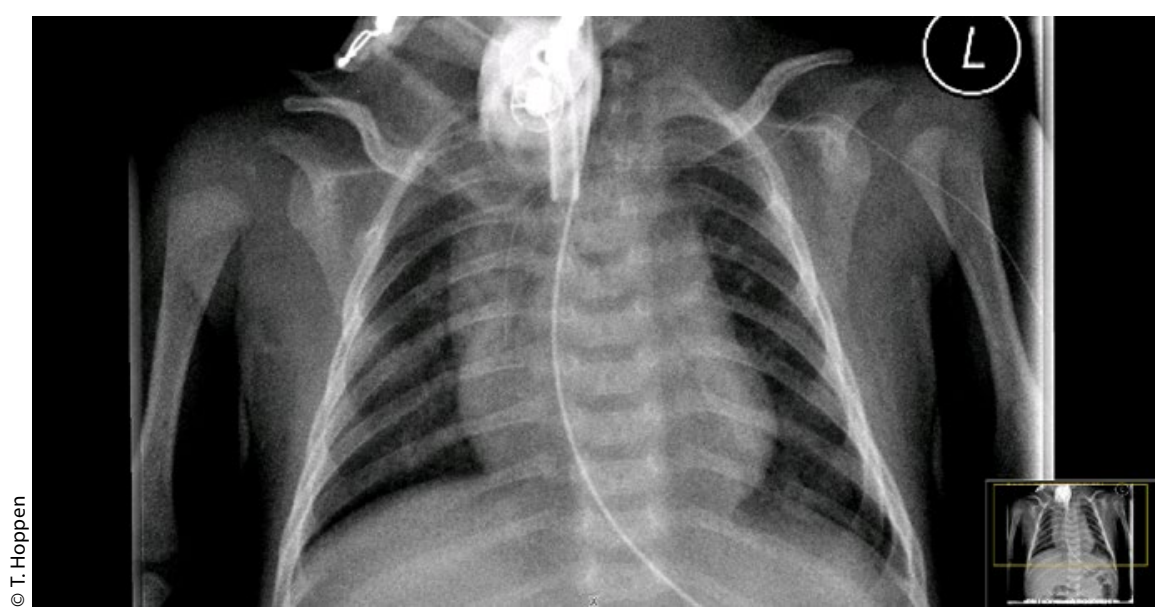

Tracheotomiertes ehemaliges Frühgeborenes mit schwierigem Atemweg (Glossoptose) im Alter von 2 Monaten

mit schwerer Lungenerkrankung betroffen. Diese Studie mit Einschluss von Neugeborenen jeglichen Reifealters bei Geburt zeigte, dass die Tracheotomierate 1997 noch bei $0,01 \%$ lag und bis 2005 auf 0,1 \% zahlenmäßig anstieg und seitdem in dieser Höhe stabil blieb.

Der zweite Beitrag, erschien zum Thema „medical progress“. Ausführlich wird ein Update zu den aktuellen Möglichkeiten nichtinvasiver kontinuierlicher CPAP-Beatmung und sich hieraus ergebender Optionen zur Prävention einer Lungenschädigung gegeben [2]. Formen optimierter invasiver Beatmung wie „volume-target ventilation“ oder „neurally adjusted ventilator assist“ sind aufwendig, teilweise sehr kostspielig und werden in absehbarer Zeit nicht in ausreichender Zahl überall verfügbar sein.

Bleibt somit derzeit neben einer perfekten Extubationspraxis nach möglichst nur kurzdauernder invasiver Beatmung, die eine Reintubation unbedingt verhindern sollte, das breite Spektrum der nichtinvasiven Beatmung im Fokus. Wie lässt sich nun ein CPAP-Ver- sagen vermeiden? Etwa durch Verwendung eines T-Stück-Beatmungsgerätes anstatt Maske-Beutel, eine optimal antizipierte „CPAP-Erfolgs-Kultur“ im gesamten Erstversorgungsteam. Zusätzlich sollten, veränderte und gut überwachte Modalitäten von initialen prolongierten Blähmanövern und moderne Methoden vorsichtiger intratrachealer Surfactantapplikation möglichst ganz ohne begleitende invasive Lungendehnung zur Anwendung kommen. Insbesondere zu den beiden letztgenannten Optionen erwarten wir in naher $\mathrm{Zu}$ kunft hoffentlich gute wissenschaftlich fundierte Belege.

1. Lee JH et al. Risk factors and in-hospital outcomes following tracheostomy in infants. J Pediatr 2016;173:39-44

2. Wright $\mathrm{CJ}$ et al. Continuous positive airway pressure to prevent neonatal lung injury: how did we get here, and how do we improve? J Pediatr 2016;173:17-24

\section{Kommentar}

Eine Tracheotomie ist glücklicherweise ein sehr seltenes Ereignis, insbesondere bei Neugeborenen. Doch die Komorbiditäten nach Tracheotomie sind zahlreich. Hierzu zählen unter anderem auch eine gestörte Mundmotorik, Dysphagie und Probleme der Sprachentwicklung. Die Kinder profitieren meist von einer regelmäßigen logopädischen Betreuung. Eine frühe Rückverlegung der künstlichen Atemwegssicherung in seine physiologische Form sollte grundsätzlich immer - vor allem aber bei primär-pulmonalen Erkrankungen - angestrebt werden.

Wichtiger als die Behandlung ihrer Folgen ist die Vermeidung der invasiven Beatmung. Eine sehr wichtige Erkenntnis! In den vergangenen Jahren hat die Neonatologie lernen müssen, dass oftmals ein "gezieltes Weniger" mehr ist als intensivmedizinischer Aktivismus. Die apparativen und manuellen Fähigkeiten haben sich für diesen Kontext sehr positiv verändert. Natürlich muss zwischen dem asphyktischen Neugeborenen am Termin, das eher einem pädiatrischen Intensivpatienten entspricht, und dem vulnerablen extremen Frühgeborenen klar differenziert werden. Aber genau diese große Gruppe besonders kleiner Patienten steht im Blickpunkt hoffnungsvoller Entwicklungen.

Dr. Thomas Hoppen 